\title{
Uma Análise Comparativa da QoS do Skype, Yahoo! Messenger e Google Talk
}

\author{
Douglas C. P. Barbosa, Marcos A. A. Gondim, Rafael D. Lins e Rafael S. de Souza
}

\begin{abstract}
Resumo - Este artigo apresenta uma análise comparativa do desempenho dos aplicativos Skype, Yahoo! Messenger e Google Talk na comunicação de voz sobre IP entre computadores através da Internet. Será avaliada a correlação entre os sinais de voz transmitidos e recebidos a partir de suas amostras no tempo e periodogramas, com o intuito de observar a fidelidade com que cada um dos aplicativos reproduz a fala e o consumo de banda decorrente do uso desses softwares para comunicação de voz sobre IP.
\end{abstract}

Palavras-Chave - VoIP, Skype, Yahoo! Messenger, Google Talk, QoS.

Abstract - This paper presents a comparative analysis of the performance of Skype, Yahoo! Messenger and Google Talk for VoIP communication between computers over the Internet. The correlation between the transmitted and received speech signals both in the time and frequency domains are analyzed. The bandwidth usage will be evaluated for each of those VoIP system. QoS.

Keywords - VoIP, Skype, Yahoo! Messenger, Google Talk,

\section{INTRODUÇÃO}

Desde o lançamento do Skype em 2003 [1], os aplicativos de VoIP têm chamado a atenção por sua rápida difusão e utilização por diversos tipos de clientes. Tal sucesso pode ser explicado porque VoIP é uma solução de fácil implementação e de baixo custo, o que agrada tanto aos usuários domésticos quanto aos empresariais. A América Latina é o destino da maior parte do tráfego de VoIP no mundo desde 2001 e o Brasil é o país onde o uso de VoIP cresce mais rapidamente, registrando, apenas no ano de 2004, um crescimento de $112 \%$ [2]. Estudos realizados em 45 países com mais de 1,5 mil profissionais de TI indicam que o mercado mundial de VoIP cresceu $152 \%$ entre os anos de 2002 e 2006. No Brasil, somente no ano de 2007, uma em cada dez empresas optará por utilizar voz sobre IP como sistema de telefonia para longas distâncias [3].

A qualidade da voz em tais aplicativos depende não somente dos CODECs utilizados, mas também de como os pacotes são roteados e encaminhados pela rede, sobretudo em momentos de congestionamento [4]. Este é um tema que tem sido alvo de inúmeros trabalhos científicos que buscam compreender o funcionamento de aplicativos para

Douglas Contente Pimentel Barbosa, Marcos Antonio Alves Gondim, Rafael Dueire Lins e Rafael Santos de Souza, Departamento de Eletrônica e Sistemas, Centro de Tecnologia e Geociências, Universidade Federal de Pernambuco, Recife, Brasil, E-mails: douglas.contente@gmail.com, marcos_aag@yahoo.com.br,rdl@ufpe.br e rafael.souza@globo.com. comunicação VoIP e analisar seu desempenho $[5,6,7,8,9,10]$. Neste artigo será apresentado um estudo comparativo da qualidade da conversação através dos aplicativos Skype, Yahoo! Messenger e Google Talk, com enfoque na fidelidade da voz recebida em relação à que foi transmitida, além de uma breve análise das características desses aplicativos no que diz respeito ao roteamento de pacotes de dados durante os processos de autenticação e estabelecimento de chamadas.

\section{Metodologia}

Os experimentos que possibilitaram a análise de desempenho de VoIP foram realizados com três dos aplicativos mais difundidos na Internet para comunicação de Voz sobre IP: Skype versão 3.1.0.152 [1], Yahoo! Messenger versão 8.1 [11] e Google Talk versão Beta [12].

Os testes consistiram na realização de chamadas VoIP entre dois computadores onde um dos interlocutores executava um arquivo de áudio padrão, cujo som era capturado pelo microfone e transmitido via aplicativo VoIP para o outro interlocutor. $\mathrm{O}$ conteúdo da chamada VoIP era gravado em arquivos de áudio no formato wave (.wav) a uma taxa de amostragem de $48 \mathrm{kHz}$ através do software Mx Skype Recorder versão 3.2.1 [13] nas duas extremidades da comunicação. Paralelamente, todos os pacotes enviados e recebidos pelos computadores durante as chamadas eram capturados pelo software Wireshark versão 0.99 .5 anunciado em [14] como substituto do Ethereal.

Para garantir uniformidade nos testes, foi utilizado um único texto em língua inglesa para gerar dois arquivos de voz sintetizada, sendo um de voz masculina e outro de voz feminina. O sintetizador utilizado foi o TTS (Text-toSpeech) da AT\&T Labs que se encontra disponível em [15]. Os arquivos gerados possuem formato wave com taxa de amostragem de $16 \mathrm{kHz}$ e duração aproximada de 20 segundos. A densidade espectral de potência estimada através dos periodogramas dos arquivos gerados é mostrada nas figuras 01 e 02. Decidiu-se utilizar arquivos de curta duração para simular trechos unidirecionais de uma conversação telefônica, viabilizando a análise através da correlação cruzada dos sinais transmitido e recebido.

Foram realizados um total de 60 chamadas VoIP, sendo 20 para cada um dos sistemas VoIP em estudo. Destes 20, 10 foram executados com uso da voz masculina e outros 10 com uso da voz feminina. Visto que se tratava de um experimento longo, e a rede poderia sofrer mudanças significativas nesse intervalo de tempo, os dados foram coletados alternando-se o aplicativo em teste. Desta forma, buscou-se minimizar a influência das oscilações nas 
condições da rede do provedor de acesso a Internet no resultado final do experimento. Por fim, cada chamada gerava 02 arquivos de áudio (transmitido e recebido) e 02 arquivos com a captura dos pacotes realizada nos computadores envolvidos no processo.

Os dois computadores estavam situados em pontos dentro da região metropolitana da cidade de Recife e ambos encontravam-se conectados a Internet através de linhas ADSL configuradas com taxas de $512 \mathrm{kB} / \mathrm{s}$ no sentido down e $128 \mathrm{kB} / \mathrm{s}$ no sentido up. Um dos computadores utilizados era um laptop Intel Centrino Duo $1.8 \mathrm{GHz}$ com $1 \mathrm{~GB}$ de RAM e o outro um PC Pentium IV $1.4 \mathrm{GHz}$ com $512 \mathrm{MB}$ de RAM.

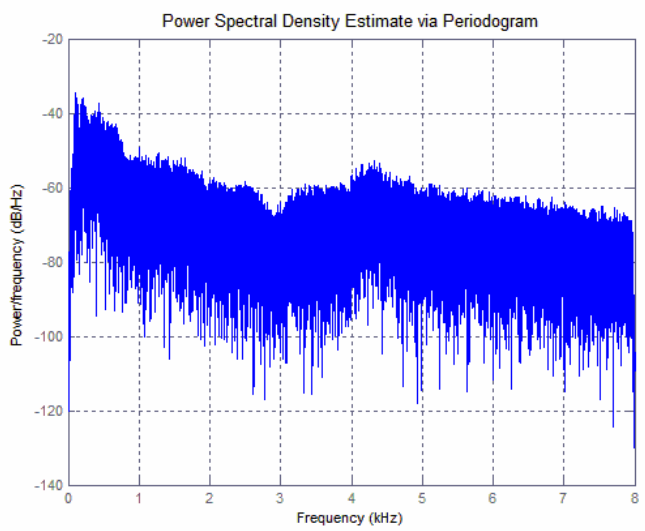

Figura 01. Densidade espectral de potência estimada via periodograma do sinal de voz masculina sintetizado.

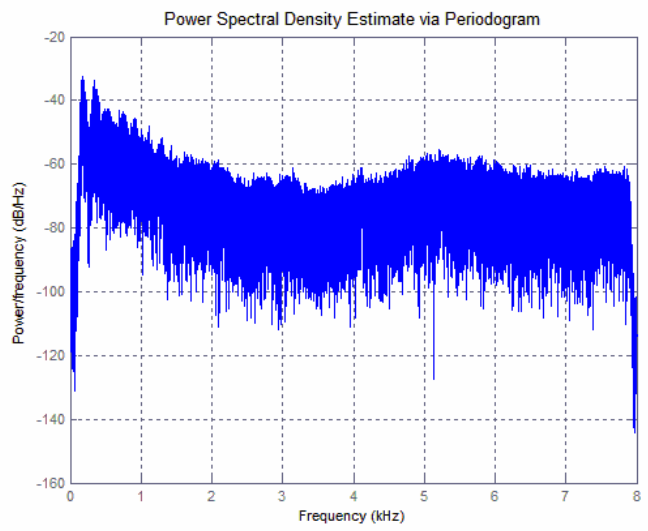

Figura 02. Densidade espectral de potência estimada via periodograma do sinal de voz feminina sintetizado.

\section{CARACTERÍSTICAS DE ROTEAMENTO}

Nesta seção serão descritos alguns aspectos importantes dos processos de autenticação e estabelecimento de chamada por cada um dos aplicativos testados bem como a topologia da rede utilizada por eles.

\section{A. Funcionamento do Skype}

De acordo com as referências [5,6,7] o Skype utiliza um esquema peer-to-peer de comunicação baseado em três entidades principais:

\section{1) Cliente Skype}

É uma aplicação Skype que pode ser utilizada para a realização de chamadas de voz, envio de mensagens de texto ou troca de arquivos.

\section{2) Super-Nó}

O Super-Nó provê à rede funcionalidades como encaminhamento de pedidos aos destinos apropriados e responder às solicitações de Clientes Skype ou de outro Super-Nó. Os Super-Nós podem ainda prover serviços de proxy para mídia de Clientes Skype que possuam acesso restrito à Internet através de NATs (Network Adress Translators) ou firewalls. Tipicamente os Super-Nós mantêm uma rede de overlay entre si enquanto um Cliente Skype liga-se com um único ou um pequeno número de Super-Nós. Uma análise de [7] mostra que um Cliente Skype pode ser promovido a Super-Nó se ele possui ampla largura de banda e é facilmente alcançável na rede.

\section{3) Servidor de Login Skype}

É o único servidor central da rede Skype. Ele é o servidor que guarda os registros de nomes dos usuários e seus passwords, realizando a autenticação dos mesmos garantindo que eles sejam únicos no espaço de nomes do Skype.

Um Cliente Skype deve se conectar a pelo menos um SuperNó e ao Servidor de Login para ser registrado na rede. O Cliente Skype possui uma lista que é atualizada periodicamente de pares endereço IP e número de porta dos Super-Nós acessíveis da rede. Essa lista, chamada segundo [5] de Host Cache, pode conter até 200 entradas. Pelo menos uma dessas entradas deve ser válida, ou seja, deve ser uma máquina on-line rodando o Skype. Após o primeiro login depois da instalação, a referência [5] observou ainda que a Host Cache é sempre inicializada com 07 entradas chamadas de Bootstrap Super Nodes. Essas entradas correspondem a Super-Nós conectados à Internet através de quatro servidores: Superb, Suscon e ev1.net nos Estados Unidos e um quarto de extensão dinamarquesa.

$\mathrm{Na}$ primeira vez que se conecta à rede, o Cliente Skype envia pacotes UDP para algum dos Bootstrap Super Nodes que o responde com outros pacotes UDP. Em seguida, o Cliente Skype estabelece com ele uma conexão TCP e provavelmente recebe o IP do Servidor de Login (80.160.91.11), com o qual estabelece uma nova conexão TCP, realiza a autenticação e finaliza a conexão TCP. A conexão TCP com o Super-Nó dura até que este se torne indisponível e quando isto ocorre o Cliente Skype inicia uma nova conexão TCP com outro Super-Nó. Nos acessos subseqüentes o procedimento é o mesmo, com exceção que ao invés de conectar um Bootstrap Super Node, o usuário pode conectar-se a qualquer Super-Nó presente na Host Cache.

Após serem ambos registrados na rede, para estabelecer uma chamada, o Cliente Skype chamador inicia uma conexão TCP com o chamado. Troca de sinalização é realizada através dessa conexão, o que indica a existência de um mecanismo de challenge-response entre os usuários. Além 
disso, pacotes UDP são enviados pelo chamador para alguns nós que estão on-line e foram descobertos durante o login. Ao ser finalizada a sinalização, inicia-se a troca de pacotes UDP de voz entre os dois usuários.

O processo descrito aqui se refere ao estabelecimento de chamadas entre dois Clientes Skype que estejam on-line com endereços IP válidos e presentes na lista de contatos um do outro, sem nenhuma restrição de NAT ou firewall. Variantes deste esquema são possíveis, mas fogem do escopo deste texto e são descritas em [5].

\section{B. Funcionamento do Yahoo! Messenger}

Através do monitoramento do tráfego de pacotes entre os usuários observa-se que o Yahoo! Messenger troca pacotes no momento da autenticação utilizando um esquema clienteservidor através do protocolo SSLv3 que provê a privacidade e a integridade de dados entre duas aplicações que se comunicam pela Internet. Isto ocorre por meio da autenticação das partes envolvidas e da criptografia dos dados transmitidos entre as mesmas. Esse protocolo ajuda a prevenir que intermediários entre as duas pontas da comunicação tenham acesso indevido ou falsifiquem os dados que estejam sendo transmitidos. A figura 03 ilustra um usuário com acesso ADSL e IP válido 201.50.209.91 efetuando a autenticação no servidor Yahoo! Messenger com IP 209.73.168.74. Utilizando-se a ferramenta My Trace Route do Fedora 6 [16], identificaram-se todos os saltos até o servidor de autenticação do Yahoo! Messenger, com estatísticas de perda e atraso dos pacotes como mostra a tabela I.

TABELA I

ESTATÍSTICA DO ROTEAMENTO DOS PACOTES DURANTE O LOGIN NO YAHOO! MESSENGER.

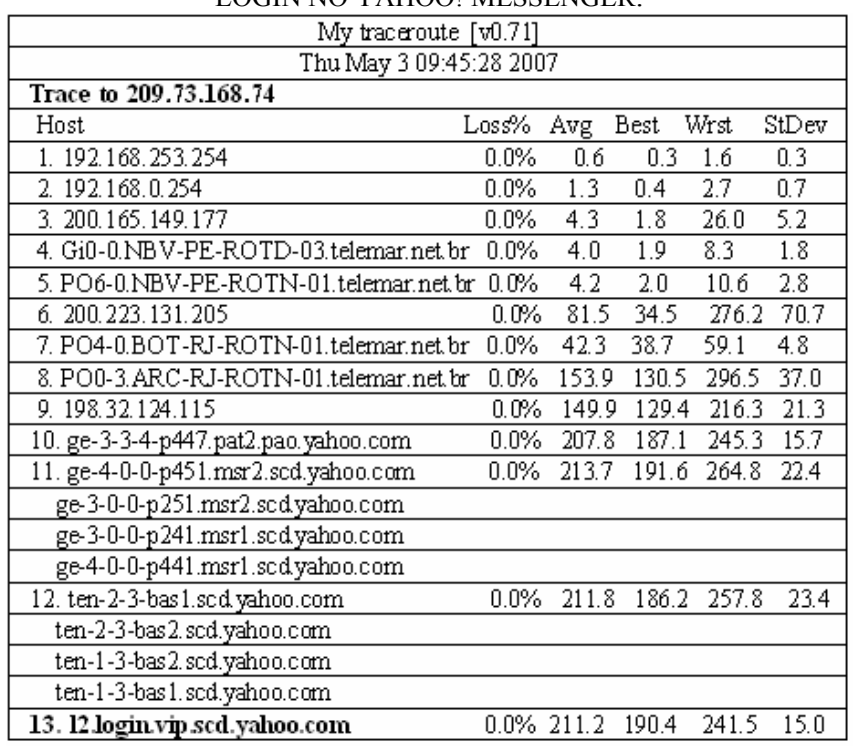

Os pacotes que foram capturados no decorrer das chamadas indicam que a partir do momento em que o usuário disca para seu destino, basicamente quatro endereços IP da rede Yahoo! foram conectados:

1. 68.142.233.145 - sip48.voice.re2.yahoo.com: servidor de sinalização SIP. Observou-se que os computadores que rodavam os aplicativos VoIP trocaram pacotes com este servidor do início ao fim da chamada;

2. 68.142.233.72 - relay1.voice.vip.re2.yahoo.com: foram observados pacotes relacionados a solicitação para ligar (Binding Request) a esse servidor;

3. 68.142.233.76 - stun2a.voice.re2.yahoo.com: o protocolo STUN (Simple Transversal of UDP Through NAT), é usado para transposição de NAT;

4. 68.142.233.79 - relay6.voice.re2.yahoo.com: servidor que é contatado no momento que antecede a inicialização da troca de pacotes de voz (UDP) entre os usuários.

A figura 04 mostra a troca de pacotes entre o usuário e os IPs dos servidores citados correspondente a somente uma das capturas realizadas, num total de 60. Embora tenha sido observada a troca de pacotes com outros endereços IP no decorrer das outras capturas, realizadas em momentos diferentes, o destino sempre correspondia a um servidor com as mesmas funcionalidades que as citadas acima.

\section{Funcionamento do Google Talk}

Analisando-se o Google Talk no momento do login, observa-se que o mesmo trabalha na forma de clienteservidor, como ilustrado na figura 05 . O IP com o qual o aplicativo troca pacotes no momento do login, é o 64.233.161.147.

TABELA II

ESTATÍSTICA DO ROTEAMENTO DOS PACOTES DURANTE O LOGIN NO GOOGLE TALK.

\begin{tabular}{|c|c|}
\hline \multicolumn{2}{|c|}{ Wy traceroute [v0.71] } \\
\hline \multicolumn{2}{|c|}{ Thu Way $313229 \cdot 502007$} \\
\hline Host & Los\% Avg Best Wrat StDiev \\
\hline 1.192 .16253 .254 & $\begin{array}{llll}0.0 \% & 0.5 & 0.4 & 09\end{array}$ \\
\hline 2.192 .1600 .254 & $\begin{array}{lll}0.0 \% & 1.2 & 0.8\end{array}$ \\
\hline 3.2010.165.149.177 & 2.5133 \\
\hline 4. GiD-0NBV-PE-ROTD-03.telemar.net br & $0.0 \%$ \\
\hline 5. PO6-0.NBV-PE-FOTN-01 telemar.net.br & $00 \%$ \\
\hline 6.2010 .223131 .205 & $\begin{array}{lllll}0.0 \% & 78.4 & 51.1 & 191.6 & 40.6\end{array}$ \\
\hline 7. PO40.BOT-RJ-ROTN-01.telemar.netbr & $\begin{array}{lllll}0.0 \% & 58.4 & 45.3 & 5.2 & 9.9\end{array}$ \\
\hline 8.200187 .12866 & $\begin{array}{llllll}0.0 \% & 191.7 & 1658 & 2283 & 24.1\end{array}$ \\
\hline 9. GigboitEthernet5-0 GWW12.VYC1 ALTERLET & $0.0 \% 3026281.8340 .7 \quad 21.4$ \\
\hline 1-DGigabitEthemet GW12.NYC1.ALTER NET & \\
\hline $10.0 .50-1-3-0 . X L 1$ VYC1 ALTER HET & $7.1 \% 305.8286 .5348 .3 \quad 20.1$ \\
\hline 11.0.so-6-1-1.XL3.VYC4ALTERHET & $0.0 \% 301.2279 .2340 .9 \quad 20.7$ \\
\hline 12.0g-5-[0-0.BR2.VYC4ALTERHET & $0.0 \% \quad 297.4278 .53376 \quad 20.1$ \\
\hline 13. Te-3-4car1.New York1 Level3.net & $0.0 \% 3763261.968891296$ \\
\hline 14. ae-1-53bbrl NewTork1 LeveB net & $0.0 \% \quad 1947 \quad 1623 \quad 2634 \quad 30.2$ \\
\hline 15. as-3-0.bbrl .Washirgtorl Level3.net & $0.0 \% 2866257.73445 \quad 24.0$ \\
\hline ae-[-0.bb2. Washirgtorl LevelBnet & \\
\hline 16. ae-21-54.carl Washingtonl Level3 net & $\begin{array}{lllll}00 \% & 2879 & 2589 & 3380 & 23.1\end{array}$ \\
\hline ae-21-52.carl. Washingtonl Level3net & \\
\hline ae-11-51.carl. Washingtord Leve13 net & \\
\hline ae-11-53 carl.Washingtonl Level3 net & \\
\hline ae-21-56.carl. Washingtorl Leve13 net & \\
\hline 17. GOOGLE-INC carl Level3 net & $\begin{array}{llllll}0.0 \% & 277.0 & 248.7 & 326.5 & 25.1\end{array}$ \\
\hline 18.72 .14238 .136 & $\begin{array}{lllll}0.0 \% & 292.2 & 262.0 & 332.4 & 23.8\end{array}$ \\
\hline 66.24995 .149 & \\
\hline 19.72 .14236 .173 &  \\
\hline 216.239 .47 .1 & \\
\hline 216.239 .49 .45 & \\
\hline 72.14 .236 .15 & \\
\hline $20.216 .230 .43 .1 \quad$ el-irifl25.google.com & $0.0 \% 292.4267 .6$ \\
\hline
\end{tabular}

Capturando-se somente os pacotes TCP através do Wireshark [14], foi observado que o Google Talk envia periodicamente pacotes para o IP 209.85.163.125 que tem como DNS el-in-f125.google.com. Através do My Trace 
Route do Fedora 6 [16] foram obtidos os saltos desde a máquina que continha o aplicativo até o servidor da Google para autenticação de usuário. O resultado obtido encontrase na tabela II.

O caminho feito desde a rede onde se encontra a máquina do usuário até o servidor Google pode ser descrita de forma resumida na seguinte seqüência de saltos:

1. 192.168.253.254, LAN onde se encontra a máquina do usuário;

2. 200.165.149.177 IP válido atrelado a LAN do usuário;

3. Estação da Telemar localizada no bairro da Boa Vista, Recife - PE;

4. Estação da Telemar localizada no bairro de Botafogo, Rio de Janeiro - RJ;

5. Rede Gigabit em Nova York DC;

6. Washington;

7. Servidor Google.

A figura 06 mostra um trecho de uma captura, de uma conversação em andamento no Google Talk. Em destaque, Na linha 41 da captura, observa-se uma mensagem "Jabber Request” (Requisição para falar), enviada para o servidor do Google Talk. Esses pacotes foram trocados antes do início da conversação. A partir da linha 49 as duas máquinas começam a trocar pacotes de voz entre si somente trocando pacotes com o servidor 209.85.163.125 em casos de retransmissão.

\section{ANÁLISES E RESUltados}

A análise dos dados foi realizada em duas etapas: uma para tratamento dos arquivos de voz gravados e identificação da correlação entre os pares de sinais (transmitido e recebido) nos domínios do tempo e da freqüência; outra para análise e determinação das características dos pacotes capturados.

Basicamente, o tratamento dado aos arquivos de áudio consistiu na normalização do tamanho de cada sinal através do corte de trechos no início e no final de cada seqüência, de forma a preservar apenas o conteúdo da fala. Tal operação foi realizada através do software Nero Wave Editor versão 3.0.0.0 [17] e tinha o intuito de viabilizar a análise comparativa entre os sinais através da correlação cruzada entre eles, além de eliminar ruídos provenientes de tons de chamada ou encerramento das ligações produzidos pelos próprios aplicativos VoIP. Os arquivos finais possuíam duração de 22 segundos e tamanho de $2.063 \mathrm{kB}$.

Foi utilizada correlação cruzada como um dos métodos para comparação entre os sinais. A função de correlação cruzada entre $f[n]$ e $g[n]$, duas funções de tempo discreto, é definida por [18]:

$$
c[m]=E\{f[n] g[n+m]\},
$$

em que $E\{X\}$ é o valor esperado da variável $X$.

Sejam $f[n]$ as amostras de voz transmitidas e $g[n]$ as amostras de voz recuperadas no receptor. A função de correlação cruzada, portanto, nos informa o quão $f[n]$ é semelhante a cópias deslocadas de $g[n]$ em função do deslocamento $m$. No caso em questão, como se espera que idealmente as amostras transmitidas e recebidas sejam iguais, se ambas as amostras possuem comprimento $N$, a função de correlação cruzada possui comprimento $2 N-1$, simetria em relação a $N$ e um máximo nas redondezas deste ponto (em função do atraso inerente a transmissão dos pacotes [19]).

Assim, através do Matlab $7^{\circledR}$, o valor máximo da função de correlação cruzada pôde então ser determinado e utilizado como um indicador de fidelidade entre o sinal de voz transmitido e o sinal recebido.

O segundo parâmetro utilizado na comparação entre os aplicativos baseou-se no cálculo da correlação entre a densidade espectral de potência dos sinais de voz transmitido e recebido, densidade esta estimada através do método de periodograma [18] e com suporte do Matlab $7^{\circledR}$. Nosso objetivo, com esses procedimentos, foi avaliar a fidelidade da reprodução do sinal de voz não apenas no domínio do tempo, mas também no domínio da freqüência. $\mathrm{Na}$ segunda etapa da análise, foram extraídas, a partir das capturas realizadas pelo software Wireshark [15], informações como jitter, perda de pacotes, tamanho médio dos pacotes transmitidos entre os computadores, taxa de envio dos pacotes e consumo de banda para cada um dos aplicativos testados. O tempo relativo de envio e chegada de cada pacote foi utilizado para estimar o valor do jitter imposto pela rede durante as chamadas, a fim de nos certificar de que os aplicativos enfrentaram em média as mesmas condições durante a bateria de testes.

$O$ fato de os relógios das máquinas não estarem sincronizados não gera problemas para o cálculo do jitter, dado que o mesmo é definido como a variância dos tempos de chegada dos pacotes enviados da máquina A para a máquina $\mathrm{B}$, e não é influenciado pelo offset inicial entre os relógios. O descompasso entre os clocks das máquinas, foi desprezado em virtude da duração das chamadas não ser suficientemente longa para que este efeito se tornasse considerável.

Não foi observada nenhuma perda de pacotes durante o experimento, o que pode ser explicado pela curta duração das chamadas - cerca de 20 segundos. Observou-se ainda que o jitter sofreu poucas variações no decorrer do ensaio como mostrado na tabela III.

TABELA III

JITTER ESTIMADO DURANTE A COMUNICAÇÃO (ms).

\begin{tabular}{|l|c|c|}
\hline Aplicativo & Média & Desvio Padrão \\
\hline Google Talk & 9,20 & 2,40 \\
\hline Skype & 7,92 & 3,03 \\
\hline Yahoo! Messenger & 8,50 & 3,17 \\
\hline
\end{tabular}

As tabelas IV e V mostram que o Skype transmitiu quase o dobro dos pacotes em cada chamada se comparado ao Google Talk e ao Yahoo! Messenger, que ficaram praticamente empatados neste item.

TABELA IV

NÚMERO DE PACOTES TRANSMITIDOS PELOS APLICATIVOS.

\begin{tabular}{|c|c|c|}
\hline Aplicativo & Média & Desvio Padrão \\
\hline Google Talk & 469,00 & 23,66 \\
\hline Skype & 830,25 & 38,07 \\
\hline Yahoo! Messenger & 453,85 & 69,85 \\
\hline
\end{tabular}


TABELA V

PACOTES TRANSMITIDOS POR SEGUNDO PELOS APLICATIVOS.

\begin{tabular}{|c|c|c|}
\hline Aplicativo & Média & Desvio Padrão \\
\hline Google Talk & 18,01 & 0,35 \\
\hline Skype & 23,59 & 1,05 \\
\hline Yahoo! Messenger & 17,87 & 2,76 \\
\hline
\end{tabular}

Não há diferenças significativas no tamanho dos pacotes gerados pelos três programas, no entanto foi observada no Yahoo! Messenger uma maior variação no tamanho desses pacotes no decorrer da transmissão.

TABELA VI

TAMANHO MÉDIO DOS PACOTES (BYTES).

\begin{tabular}{|c|c|c|}
\hline Aplicativo & Média & Desvio Padrão \\
\hline Google Talk & 172,95 & 2,19 \\
\hline Skype & 160,91 & 1,97 \\
\hline Yahoo! Messenger & 188,83 & 22,27 \\
\hline
\end{tabular}

Quanto à taxa de transmissão, é mostrado na tabela VII que o Skype apresentou em média um maior consumo de banda que os outros dois aplicativos, custo esse que pode estar relacionado ao o uso de piggybacking.

TABELA VII

TAXA DE TRANSMISSÃO (kB/s).

\begin{tabular}{|c|c|c|}
\hline Aplicativo & Média & Desvio Padrão \\
\hline Google Talk & 3,11 & 0,08 \\
\hline Skype & 3,78 & 0,18 \\
\hline Yahoo! Messenger & 3,36 & 0,48 \\
\hline
\end{tabular}

As tabelas VII, IX, X e XI exibem os resultados obtidos no cálculo da correlação entre os sinais de voz transmitido e recebido nos domínios do tempo e da freqüência, de acordo com os métodos expostos na seção IV.

TABELA VIII

CORRELAÇÃO MÁXIMA ENTRE OS PERIODOGRAMAS DOS ARQUIVOS DE VOZ TRANSMITIDOS E RECEBIDOS.

\begin{tabular}{|c|c|c|}
\hline Aplicativo & Média & Desvio Padrão \\
\hline Google Talk & 0,243 & 0,027 \\
\hline Skype & 0,215 & 0,036 \\
\hline Yahoo! Messenger & 0,210 & 0,033 \\
\hline
\end{tabular}

TABELA IX

CORRELAÇÃO MÁXIMA ENTRE OS SINAIS DE VOZ.

\begin{tabular}{|c|c|c|}
\hline Aplicativo & Média & Desvio Padrão \\
\hline Google Talk & 0.164 & 0.054 \\
\hline Skype & 0.188 & 0.095 \\
\hline Yahoo! Messenger & 0.158 & 0.038 \\
\hline
\end{tabular}

TABELA X

CORRELAÇÃO MÁXIMA ENTRE OS SINAIS DE VOZ MASCULINA.

\begin{tabular}{|c|c|c|}
\hline Aplicativo & Média & Desvio Padrão \\
\hline Google Talk & 0,188 & 0,064 \\
\hline Skype & 0,217 & 0.113 \\
\hline Yahoo! Messenger & 0,146 & 0,030 \\
\hline
\end{tabular}

TABELA XI

CORRELAÇÃO MÁXIMA ENTRE OS SINAIS DE VOZ FEMININA.

\begin{tabular}{|c|c|c|}
\hline Aplicativo & Média & Desvio Padrão \\
\hline Google Talk & 0,140 & 0,031 \\
\hline Skype & 0,159 & 0,066 \\
\hline Yahoo! Messenger & 0,170 & 0,043 \\
\hline
\end{tabular}

\section{CONCLUSÕES E TRABALHOS Futuros}

Os sistemas de comunicação de voz sobre IP tornaram-se muito populares nos últimos anos. Poucos estudos comparativos como o realizado em [9] são encontrados na literatura, apesar da enorme quantidade de usuários que utilizam esses sistemas. A tabela VIII nos mostra que a codificação PCM de taxa de bit variável utilizada pelo Google Talk consegue manter uma maior fidelidade entre os espectros dos sinais transmitidos e recebidos em relação à atingida pelo codificador iLBC [20] do Skype. Os valores observados para o Yahoo! Messenger indicam que esse aplicativo deve utilizar uma estratégia de codificação similar à utilizada pelo Skype. As tabelas IX, X e XI mostram, no entanto, que em média a fidelidade dos sinais no Skype é ligeiramente superior às dos outros dois aplicativos sofrendo, porém, oscilações maiores, provavelmente devido à estratégia de play-out FIFO (Firt-in-first-out) tornando-o mais dependente das condições da rede, já que os pacotes que chegam desordenados são reproduzidos nesta mesma ordem [9].

O Skype apresentou um maior consumo de banda durante a realização das chamadas enquanto o Yahoo! Messenger e Google Talk mostraram características bastante semelhantes no que diz respeito a desempenho, número e tamanho dos pacotes transmitidos entre os computadores e largura de banda utilizada. Em trabalhos futuros pretende-se buscar uma maior compreensão das diferenças entre os aplicativos e seus métodos de codificação e transmissão de voz, acrescentando outras formas de comparação dos arquivos de áudio, tanto quantitativas quanto subjetivas, correlacionando os resultados aqui apresentados com os obtidos pelos métodos já existentes para a qualificação de voz, como o MOS (Mean Opinion Score). É intensão deste estudo, repetir os experimentos com enlaces intercontinentais e com chamadas de maior duração também pode trazer à tona novas características a serem analisadas. Pretende-se também comparar os aspectos da transmissão conjunta de voz e vídeo na Internet, a degradação dos sinais de voz trafegando em meios sem-fio nas extremidades do circuito de comunicação (rede $802.11+$ rede cabeada + rede 802.11), e a qualidade da voz em ligações entre VoIP e telefones TDM. 


\section{REFERÊNCIAS}

[1] Skype: http://www.skype.com/

[2] Telegeography: http://www.telegeography.com/press/releases/200512-15.php. Acessado em 30/04/2007.

[3] Estadão:.http://www.estadao.com.br/tecnologia/internet/noticias/2006 /dez/14/228.htm. Acessado em 30/04/2007.

[4] William C. Hardy, "VoIP Service Quality - Measuring and Evaluating Packet-Switched Voice", McGraw-Hill Book Co, 2003.

[5] S. A. Basset., H. Schulzrinne, "An Analysis of the Skype Peer-to-Peer Internet Telephony Protocol", Proceedings of IPTPS, Dec. 5th, 2004.

[6] S. Ehlert, S. Petgang, "Analysis and Signature of Skype VoIP Session Traffic", Proceedings of Communications, Internet and Information Technology, July 25th, 2006.

[7] S. Guha, N. Daswani, R. Javi, "An Experimental Study of the Skype Peer-to-Peer VoIP System”, Proceedings of IPTPS, 2006.

[8] T. Hoßfeld, A. Binzenhöfer, M. Fiedler, K. Tutschku, "Measurement and Analysis of Skype VoIP Traffic in 3G UMTS Systems", Research Report Series, University of Würzburg, December 2005.

[9] S. Batu, B. W. Wah, "Analysis and Evaluation of the Skype and Google-Talk Voip Systems", IEEE International Conference on Multimedia and Expo, July 2006.
[10] H.Nishida and T.Suzuki, "Performance Analysis of a P2P-Based VoIP Software", International Conference on Internet and Web Applications and Services/Advanced International Conference on Telecommunications, February 2006.

[11] Yahoo Messenger: http://messenger.yahoo.com/

[12] Google Talk: http://www.google.com/talk/

[13] Mx Skype Recorder: http://www.skyperec.com/

[14] Wireshark: http://www.wireshark.org/

[15] TTS: http://www.research.att.com/ ttsweb/tts/demo.php. Acessado em 30/04/2007.

[16] My Trace Route: http://fedoraproject.org/wiki/

[17] Nero: www.nero.com

[18] A. V. Oppenheim, R. W. Schaffer, "Discrete-Time Signal Processing”, Prentice-Hall, 1989.

[19] O. Hersent, J.P. Petit, D. Gurle, "Beyond VoIP Protocols Understanding Voice Technology and Networking Techniques for IP Telephony", John Wiley \& Sons, 2005.

[20] S. Andersen et. al, "Internet Low Bit Rate Codec (iLBC)", http://www.ietf.org/rfc/rfc3951.txt, December, 2004.

\begin{tabular}{|c|c|c|c|c|c|}
\hline 2114.109223 & 201.50 .209 .91 & 209.73 .168 .74 & TCP & $1529>$ https $[5 Y N]$ Seq $=0$ Len $=0$ MSS $=1360$ & 7014.109223 \\
\hline 2214.234835 & 201.50 .209 .91 & 216.155 .193 .150 & TCP & $1528>5050 \quad[A C K]$ Seq $=56$ ACK $=140$ win $=65396$ Len: & 6214.234835 \\
\hline 2314.569939 & 209.73 .168 .74 & 201.50 .209 .91 & Ten & 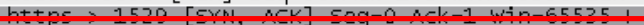 & 6814.569939 \\
\hline 2414.571738 & 201.50 .209 .91 & 209.73 .168 .74 & TCP & $1529>$ https $[\mathrm{ACK}]$ Seq $=1$ ACK $=1$ win $=65535$ Len $=0$ & 6214.571738 \\
\hline 2514.572514 & 201.50 .209 .91 & 209.73 .168 .74 & SSLV3 & Client Hello & 16414.572514 \\
\hline 2815.102690 & 209.73 .168 .74 & 201.50 .209 .91 & SSLV3 & Server Hel1o, Certificate, Server Hel & 91815.102690 \\
\hline 3015.595401 & 209.73 .168 .74 & 201.50 .209 .91 & SSLV3 & Change Cipher spec, server He17o[Malformed Pacl & 12915.595401 \\
\hline 3115.598592 & 201.50 .209 .91 & 209.73 .168 .74 & SSLV3 & Application Data & 63915.598592 \\
\hline 3216.186567 & 209.73 .168 .74 & 201.50 .209 .91 & SSLV3 & application Data & 119816.186567 \\
\hline 3316.188486 & 201.50 .209 .91 & 209.73 .168 .74 & TLF & $1329>\operatorname{ilcps}[\mathrm{F} 1 \mathrm{~N}, \mathrm{ACK}] \mathrm{Seq}=884$ ACK $=2$ & 6216.188486 \\
\hline 3416.189709 & 201.50 .209 .91 & 216.155 .193 .150 & TCP & [TCP segment of a reassembled PDU] & 88916.189709 \\
\hline
\end{tabular}

Figura 03. Captura dos pacotes durante a autenticação do Yahoo! Messenger.

\begin{tabular}{|c|c|c|c|c|c|c|c|}
\hline & 0.438477 & 201.32 .165 .30 & 68.142 .233 .145 & TCP & ttps [ACK] Seq=497 ACk $=1157$ win $=16123$ । & 54 & 0.438477 \\
\hline & 0.438477 & 01.32 .165 .30 & 68.142 .233 .145 & SSL & Continuation Data & 570 & 0.438477 \\
\hline 6 & 0.447266 & 201.32 .165 .30 & 68.142 .233 .72 & TCP & $1782>$ https [SYN] Seq $=0$ Len $=0$ MSS $=1440$ & 62 & 0.447266 \\
\hline & 0.861328 & 8.142 .233 .72 & 201.32 .165 .30 & TCP & https $>1782$ [SYN, ACK $]$ Seq $=0$ ACK $=1$ win $=65535$ । & 60 & 0.861328 \\
\hline 8 & 0.861328 & 1.32 .165 .30 & 68.142 .233 .72 & TCP & $1782>$ https [ACK] Seq $=1$ ACK $=1$ win $=17280$ Len $=0$ & 54 & 0.861328 \\
\hline 9 & 0.861328 & $=165$ & 68.142 .233 .72 & SSL & Continuation Data & 174 & 0.861328 \\
\hline 10 & 0.945313 & .142 .233 .145 & 201.32 .165 .30 & $\mathrm{TCP}$ & https > 1768 [ACK] seq $=1157$ ACK $=1013$ win $=65535$ & 60 & 0.945313 \\
\hline 11 & 1.269532 & .142 .233 .72 & 201.32 .165 .30 & SSL & Continuation Data & 86 & 9532 \\
\hline 12 & 1.269532 & 201.32 .165 .30 & 68.142 .2 & TCP & $1782>$ https $[$ FIN, ACK $]$ seq $=121$ ACK $=33$ win $=172$ & 54 & 532 \\
\hline 13 & 1.276368 & 201.32 .165 .30 & 200.165 .1 & DNS & standard query A stun. voice. bro. yahoo. com & 84 & 6368 \\
\hline 14 & 1.3 & .155 & 201 & DNS & standard query response CNAME stun. voice.yahoo: & 158 & L96 \\
\hline 15 & 1.3 & .30 & 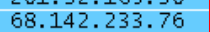 & STUN & Message: Binding Request & 70 & 96 \\
\hline 16 & 1.3 & 201. & 68. & TCP & $1783>$ https $[S Y N]$ Seq $=0$ Len $=0$ MSS $=1440$ & 62 & 25 \\
\hline 17 & 1.3 & 30 & 68. & STUN & Message: Binding Request & 70 & \\
\hline 18 & 1.3 & 201 & 68. & TCP & $1784>$ https $[S Y N]$ Seq $=0$ Len $=0$ MSS $=1440$ & 62 & 961 \\
\hline 19 & & & & UDP & source port: 8009 Destination port: 13844 & 154 & \\
\hline 20 & 1.3 & 201. & 68. & UDP & Source port: 8010 Destination port: 13845 & 154 & 914 \\
\hline 21 & 1.4 & & 68. & STUN & Message: Binding Request & 70 & \\
\hline 22 & 1.4 & 201.32 .16 & 68. & STUN & Message: Binding Request & 70 & 7266 \\
\hline 23 & 1.6 & 201.3 & 68. & STUN & Message: Binding Request & 70 & 1.634766 \\
\hline 24 & 1.647461 & 201.32 .165 .30 & 68.142 .233 .76 & STUN & Message: Binding Request & 70 & 1.647461 \\
\hline
\end{tabular}

Figura 04. Troca de pacotes entre o usuário e o servidor no Yahoo! Messenger.

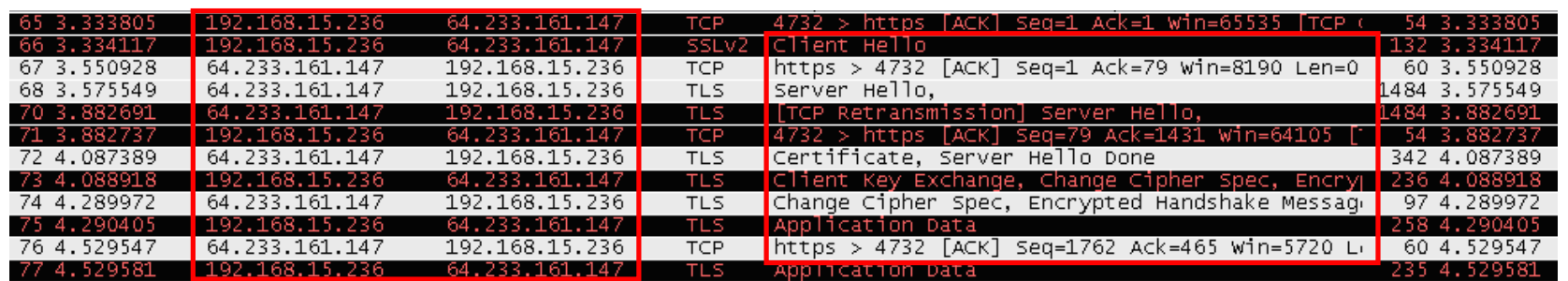

Figura 05. Captura de pacotes durante a autenticação do Google Talk.

\begin{tabular}{|c|c|c|c|c|c|}
\hline 415.341797 & 201 & -63.125 & Jabber & Request: $\backslash 02$ & \\
\hline 425.343750 & 53.125 & 1.32 .165 .30 & Jabber & Response: $\backslash 027 \backslash 003 \backslash 001 \backslash 000 \backslash 210 \mathrm{P}$ & 195 \\
\hline $43 \quad 5.347656$ & .163 .125 & 1.32 .165 .30 & Jabber & $027 \backslash 003 \backslash 001 \backslash 000 \backslash 210 h \backslash 215 . z \backslash 213 \backslash 2110^{\prime}$ & 1955 \\
\hline 445. & & 9.85 .163 .125 & & $1585>5222[\mathrm{ACK}]$ seq $=3646 \quad \mathrm{ACK}=1364$ win $=16170$ । & 54 \\
\hline $45 \quad 5.5$ & 125 & 20 & Jabber & $003 \backslash 001 \backslash 000 \backslash 210 \backslash 250] B \backslash 026 ? v \# \backslash 331$ & 195 \\
\hline 465.6 & .125 & 30137.16530 & TCP & K] Seq $=1505$ ACK $=3646$ win $=18720$ । & 60 \\
\hline 475. & & & $T C P$ & Seq $=3646$ Ack $=1505$ win=16029 । & 54 \\
\hline & 189 & 20 & UDP & Source port: 64904 Destination port: 53379 & 124 \\
\hline 496.9 & 18 & 201.32 .165 .30 & STUN & Message: Binding Request & 986.967773 \\
\hline $50 \quad 6.967773$ & 201.32 .165 .30 & 189.13 .172 .233 & STUN & ing Error Response & $123 \quad 6.96777$ \\
\hline
\end{tabular}

Figura 06. Captura de pacotes ligeiramente antes do início da conversação no Google Talk. 